ANNALES

POLONICI MATHEMATICI

XLVI (1985)

\title{
A general description of the Bergman projection
}

\author{
by Maciej Skwarczyński (Warszawa)
}

Franciszek Leja in memoriam

\begin{abstract}
Let $D \subset C^{N}$ be the union of domains $D_{i}, i=1,2, \ldots, m$. We shall apply a theorem of I. Halperin to describe the Bergman projection $P: L^{2}(D) \rightarrow L^{2} H(D)$ in terms of the Bergman projections in domains $D_{i}$. This yields a more constructive description of distances related to the Bergman function. A number of related recent results is reported.
\end{abstract}

1. Orthogonal projections. The following theorem is due to I. Halperin [2]. A very elegant proof, given by Amemiya and Ando, can be found in Helgason book [3], p. 55.

Theorem 1. Let $H$ be a Hilbert space, and $P_{i}$ the orthogonal projection onto the closed linear subspace $F_{i}, i=1,2, \ldots, m$. Then for every $f \in H$

$$
\lim _{n \rightarrow \infty}\left(P_{m} P_{m-1} \ldots P_{1}\right)^{n} f=P f
$$

where $P$ denotes the orthogonal projection onto $F=\bigcap_{i=1}^{m} F_{i}$.

From the above result easily follows that the sequence of alternating projections

$$
f_{1}=P_{1} f, \quad f_{2}=P_{2} f_{1}, \quad \ldots, \quad f_{m}=P_{m} f_{m-1}, f_{m+1}=P_{1} f_{m},
$$

converges in $H$ to $P f$. We shall also need a well-known theorem:

THEOREM 2. Let $H$ be a Hilbert space, and $P_{j}: H \rightarrow F_{j}, j=1,2, \ldots a$ sequence of orthogonal projections, such that $F_{1} \supset F_{2} \supset F_{3} \supset \ldots$ Then for every $f \in H$

$$
\lim _{j \rightarrow \infty} P_{j} f=P f
$$

where $P$ denotes the orthogonal projection onto $F=\bigcap_{j=1}^{\infty} F_{j}$.

For the proof see M. H. Stone [14], p. 74. 
2. Consequences for the Bergman projection. We shall consider the following cases

$1^{\circ} D \subset C^{N}$ is a finite union of domains $D_{i} \subset C^{N}, i=1,2, \ldots, m$;

$2^{\circ} D \subset C^{N}$ is the union of an increasing sequence of domains $G_{j} \subset C^{N}$, $j=1,2, \ldots$.

In the first case we can prove

TheOREM 3. Let $D \subset C^{N}$ be the union of domains $D_{i} \subset C^{N}$, $i=1,2, \ldots, m$. In the Hilbert space $H=L^{2}(D)$, consider for each $i$ the closed linear subspace $F_{i}$ of functions which are holomorphic in $D_{i}$ and arbitrary in $D \backslash D_{i}$. Let $Q_{i}: L^{2}\left(D_{i}\right) \rightarrow L^{2} H\left(D_{i}\right)$ be the Bergman projection in $D_{i}$. Then the orthogonal projection $P_{i}: H \rightarrow F_{i}$ is given by

$$
\left(P_{i} f\right)(z)= \begin{cases}\left(Q_{i} f_{\mid D_{i}}\right)(z), & z \in D_{i}, \\ f(z), & z \in D \backslash D_{i},\end{cases}
$$

and for every $f \in L^{2}(D)$

$$
\lim _{n \rightarrow \infty}\left(P_{m} P_{m-1} \ldots P_{1}\right)^{n} f=P f
$$

where $P$ is the Bergman projection in $D$.

Proof. The right-hand side of (1) is obviously in $F_{i}$. Let us denote it by g. The difference $f-g$ is in $F_{i}^{\perp}$. Indeed, it vanishes on $D \backslash D_{i}$, and on $D_{i}$ it is orthogonal to $L^{2} H\left(D_{i}\right)$. Hence, for every $h \in F_{i}$,

$$
\int_{D}(f-g) \bar{h}=\int_{D \backslash D_{i}}(f-g) \bar{h}+\int_{D_{i}}(f-g) \bar{h}=0 .
$$

This proves (1). By Halperin's theorem (2) holds where $P$ is defined as the orthogonal projection onto $F=\bigcap_{i=1}^{m} F_{i}$. Since $F=L^{2} H(D)$, we see that $P$ is the Bergman projection in $D$.

In the second case we can state

TheOREM 4. Let $D \subset C^{N}$ be the union of an increasing sequence of domains $G_{j} \subset C^{N}, j=1,2, \ldots$ Denote by $F_{j}$ the closed linear subspace in $H$ $=L^{2}(D)$ consisting of functions which are holomorphic in $G_{j}$, and arbitrary in $D \backslash G_{j}$. Let $P_{j}: H \rightarrow F_{j}$ be the corresponding orthogonal projection. Then for every $f \in L^{2}(D)$

$$
\lim _{j \rightarrow \infty} P_{j} f=P f
$$

where $P$ is the Bergman projection in $D$.

Proof. Note that subspaces $F_{j}, j=1,2, \ldots$, form a decreasing sequence. By Theorem 2 we have (3) with $\boldsymbol{P}$ defined as the orthogonal projection onto $F=\bigcap_{j=1}^{\infty} F_{j}$. Since $F=L^{2} H(D)$, we see that $P$ is the Bergman projection in $D$. 
Theorems 3 and 4 show that "in principle" we can determine the Bergman projection in an arbitrary domain $D \subset C^{N}$. Indeed, it is easy to represent $D$ as a union of an increasing sequence of domains $G_{j}$, where every $G_{j}$ is a finite union of open balls. The Bergman projection in each ball is known explicite. Therefore by Theorem 3 we can determine the Bergman projection in every $G_{j}$. Then using Theorem 4 we can determine the Bergman projection in $D$. (It may be convenient sometimes to replace balls by other domains for which the Bergman function is known explicite.)

The Bergman projection $P: L^{2}(D) \rightarrow L^{2} H(D)$ can be applied to determine the Bergman function $K_{D}(z, t), z, t \in D$. (A standard reference for the Bergman function is [1].) For an arbitrary point $t \in D$ we can find a ball $B \subset D$ with center at $t$. Then $\varphi=(\operatorname{vol} B)^{-1} \chi_{B} \in L^{2}(D)$ has the reproducing property

$$
\int_{D} f(z) \overline{\varphi(z)} d m(z)=f(t), \quad f \in L^{2} H(D) .
$$

It follows that $P \varphi$ has the same reproducing property. Since $P \varphi \in L^{2} H(D)$, we conclude that $P \varphi=K_{D}(\cdot, t)$. If we know that a sequence of operators $P_{n}$ in $L^{2}(D)$ converges pointwise to $P$ we can conclude that the sequence $\varphi_{n}$ $=P_{n} \varphi$ converges in $L^{2}(D)$ to $K_{D}(\cdot, t)$. Conversely, the Bergman function determines the Bergman projection by the formula

$$
(P f)(t)=\int_{D} f(z) \overline{K_{D}(z, t)} d m(z) .
$$

It should be noted that Theorem 4 is essentially equivalent to a theorem of I. P. Ramadanov, proved by different method in [7].

The results of this section indicate a part of the borderland in the theory of operators in Hilbert space, and the theory of several complex variables, which, in the authors opinion, will be important for the future development of both the subjects.

3. The orthogonality condition. Unfortunately we are so far not able to obtain new expressions for the Bergman function in a closed form using the procedure of Theorems 3 and 4 . Nevertheless, since the procedure is both constructive and general, we shall study it for its own sake. As a very simple example we can consider the decomposition of $C$ as a union of domains $D_{1}$, $D_{2}$ such that the sets int $D_{1}^{c}$, int $D_{2}^{c}$ are nonvoid. We shall say that $D_{1}$ and $D_{2}$ satisfy the orthogonality condition if

$$
\int_{D_{1} \cap D_{2}} h_{1} h_{2}=0 \quad \text { for every } h_{1} \in L^{2} H\left(D_{1}\right) \text { and } h_{2} \in L^{2} H\left(D_{2}\right) \text {. }
$$

It was observed in [13] that all computations required in Theorem 3 can be carried out explicitely in two simple cases: $1^{\circ}$ domains bounded by concentric circles $\left(D_{2}\right.$ is a disc and $D_{1}$ is the exterior of a smaller concentric disc); $2^{\circ}$ domains bounded by parallel lines ( $D_{1}$ and $D_{2}$ are halfplanes, which 
intersect along a strip). Since the subspaces $F_{1}, F_{2}$ in $L^{2}(C)$ (notation of Theorem 3) intersect only at 0 , we may introduce the angle $\alpha \in\left[0, \frac{1}{2} \pi\right]$ such that

$$
\cos \alpha=\sup \left\{\frac{\left|\left\langle f_{1}, f_{2}\right\rangle\right|}{\left\|f_{1}\right\| \cdot\left\|f_{2}\right\|} ; f_{i} \in F_{i} \backslash\{0\}, i=1,2\right\} .
$$

Let us associate with every domain $G \subset C^{N}$ and every measurable subset $T \subset G$ the biholomorphic invariant

$$
p_{G}(T)=\sup \left\{\frac{\|h\|_{T}}{\|h\|_{G}} ; h \in L^{2} H(G) \backslash\{0\}\right\} .
$$

Obviously, $p_{G}(T) \in[0,1]$. Write $p_{1}=p_{D_{1}}\left(D_{1} \backslash D_{2}\right), p_{2}=p_{D_{2}}\left(D_{2} \backslash D_{1}\right)$. It was proved in [9] that (4) implies $\cos \alpha=\max \left(p_{1}, p_{2}\right)$. It follows that $\alpha>0$ if domains are bounded by concentric circles, and $\alpha=0$ if domains are bounded by parallel lines. The situation where $\alpha=0$ is "not favourable" for Theorem 3. (This can be understood by looking at Halperins theorem in a finite dimensional space.) It is therefore remarkable that the convergence in case $2^{\circ}$ is still satisfactory (comparable with $\sum_{n=1}^{\infty} n^{-2}$ ).

4. Distances related to the Bergman function. The invariant distance

$$
\varrho_{D}(z, t)=\left(1-\left(\frac{K_{D}(z, t) K_{D}(t, z)}{K_{D}(z, z) K_{D}(t, t)}\right)^{1 / 2}\right)^{1 / 2}
$$

explicitely expressed by the Bergman function in a domain $D \subset \subset C^{N}$ was discovered by the author and investigated in [10]. (See also [1], p. 193.) The direct connection of $\varrho_{D}$ with the Bergman function $K_{D}$ is important for applications ([10], [11], p. 31). (See also [11], p. 25, Theorem III.15, and [6].) In a less explicite form, the distance $\varrho_{D}$ has appeared already in [4]. In a special case of the unit disc the distance $\varrho_{D}$ (in still another form) was considered by Tsuji [15].

Expression (6) defines the distance function also for some unbounded domains. A complete characterization of such domains for $n>1$ remains an open problem. For $n=1$ it is known [12] that $\varrho_{D}$ defines a distance function in $D$ if and only if $C \backslash D$ is not polar. (See also [9].)

The results of Section 2 can be used to determine $\varrho_{D}(D \subset \subset C$ for simplicity) in the following way:

Corollary 1. Assume that $\psi_{m} n=1,2, \ldots$, converges in $L^{2}(D)$ to $K_{D}(\cdot, z)$, and that $\varphi_{n}, n=1,2, \ldots$, converges in $L^{2}(D)$ to $K_{D}(\cdot, t)$. Then

$$
\varrho_{D}(z, t)=\lim _{n \rightarrow \infty}\left(1-\frac{\left|\left\langle\psi_{n}, \varphi_{n}\right\rangle\right|}{\left\|\psi_{n}\right\| \cdot\left\|\varphi_{n}\right\|}\right)^{1 / 2}
$$


Let us remark, that $\varrho_{D}$ is related to the Bergman metric

$$
d s^{2}=\sum_{j, k=1}^{N}\left(\frac{\partial^{2} \log K_{D}(z, z)}{\partial z_{j} \partial \bar{z}_{k}} d z_{j} \otimes d \bar{z}_{k}+\frac{\partial^{2} \log K_{D}(z, z)}{\partial \bar{z}_{j} \partial z_{k}} d \bar{z}_{j} \otimes d z_{k}\right)
$$

in the following way. Denote by $l_{B}(\gamma)$ the length of a $C^{1}$ curve $\gamma:[a, b] \rightarrow D$ with respect to the Bergman metric. Let us introduce the $\varrho_{D}$-length of $\gamma$ by the formula

$$
l_{e_{D}}(\gamma)=\sup \sum_{i=1}^{n-1} \varrho_{D}\left(\gamma\left(t_{i}\right), \gamma\left(t_{i+1}\right)\right) \text {, }
$$

where $a=t_{0}<t_{1}<\ldots<t_{n}=b$ are arbitrary points on the segment $[a, b]$. It was proved recently [5] that $l_{B}(\gamma)=2 l_{Q D}(\gamma)$.

5. Acknowledgement. I would like to thank Professor Krzysztof Maurin for helpful discussions during the preparation of the paper.

\section{References}

[1] S. Bergman, The Kernel Function and Conformal Mapping, Math. Surveys No. 5, Amer. Math. Soc. second ed., Providence, R.1., 1970.

[2] I. Halperin, The product of projection operators, Acta Sci. Math. (Szeged) 23 (1962), 96 99.

[3] S. Helgason, The Radon Transform, Birkhäuser 1980.

[4] S. Kobay as hi, Geometry of bounded domains, Trans. Amer. Math. Soc. 92 (1959), 267290.

[5] T. Mazur, P. Pflug, M. Skwarczyński, Invariant distances related to the Bergman funcrion, Proc. Amer. Math. Soc. 94 (1985), 72-76.

[6] T. Ohsawa, A remark on the completeness of the Bergman metric, Proc. Japan Acad. 57 (1981), 238-240.

[7] I. P. Ramadanov, Sur une propriété de la fonction de Bergman, C. R. Acad. Bulg. Sci. 20 (1967), 759-762.

[8] -, M. Skwarczyński, An angle in $L^{2}(C)$ determined by two plane domains, Bull. Polish Acad. Sci. 32 (1984), 653-659.

[9] J. Siciak, On removable singularities of $L^{2}$-holomorphic functions of several complex variables, In: Prace matematyczno-fizyczne, Wyższa Szkoła Inżynierska, Radom 1982.

[10] M. Skwarczynski, The invariant distance in the theory of pseudoconformal transformations and the Lu Qi-keng conjecture, Proc. Amer. Math. Soc. 22 (1969), 305-310.

[11] -, Biholomorphic invariants related to the Bergman functions, Dissertationes Math. 173, P.W.N., Warszawa 1980, 1-64.

[12] -, Evaluation functionals on spaces of square integrable holomorphic functions, In: Prace matematyczno-fizyczne, Wyższa Szkoła Inżynierska, Radom 1982.

[13] -, Alternating projections in complex analysis, Proceedings of the II-nd International Conference on Complex Analysis and its Applications, Varna, May 2-9, 1983, 192-199.

[14] M. H. Stone, Linear Transformations in Hilbert Space and their Application to Analysis, Amer. Math. Soc. 9-th ed. Providence, R.I, 1979.

[15] M. Tsuji, Potential Theory in Modern Function Theory, Maruzen Co,, Tokyo 1959. 